\title{
Dithiolethione ACDT Suppresses Neuroinflammation and Ameliorates Disease Severity in Experimental Autoimmune Encephalomyelitis
}

Ping-Chang Kuo ${ }^{1}$, Dennis A. Brown ${ }^{2}$, Barbara A. Scofield ${ }^{1}$, Hallel C. Paraiso ${ }^{3}$, Pei-Yu Wang ${ }^{4}$, I- $^{-}$ Chen $\mathrm{Yu}^{3}$, and Jui-Hung Yen ${ }^{1 \#}$

${ }^{1}$ Department of Microbiology and Immunology, ${ }^{3}$ Department of Anatomy and Cell Biology, Indiana University School of Medicine, Fort Wayne, IN. ${ }^{2}$ Department of Pharmaceutical Sciences, Manchester University College of Pharmacy, Natural and Health Sciences, Fort Wayne, IN. ${ }^{4}$ Graduate Institute of Brain and Mind Science, College of Medicine, National Taiwan University, Taipei, Taiwan

${ }^{\#}$ Corresponding author: Jui-Hung Yen, Ph.D. Department of Microbiology and Immunology, Indiana University School of Medicine, Fort Wayne, IN 46805. Email address: jimyen@iu.edu. Phone: 260-481-0452.

\section{Conflict of Interest Statement}

All authors declare that there are no conflicts of interest.

\section{Short title}

Dithiolethione ACDT suppresses neuroinflammation in EAE

This is the author's manuscript of the article published in final edited form as:

Kuo, P.-C., Brown, D. A., Scofield, B. A., Paraiso, H. C., Wang, P.-Y., Yu, I.-C., \& Yen, J.-H. (2018). Dithiolethione ACDT suppresses neuroinflammation and ameliorates disease severity in experimental autoimmune encephalomyelitis. Brain, Behavior, and Immunity. https://doi.org/10.1016/j.bbi.2018.03.010 


\section{Abstract}

Multiple sclerosis (MS) is an autoimmune disorder characterized by the central nervous system (CNS) infiltration of myelin-specific pathogenic $\mathrm{T}$ cells followed by brain inflammation in association with demyelination. Similarly, experimental autoimmune encephalomyelitis (EAE), the animal model of MS, also exhibits increased CNS infiltration of pathogenic T cells, including Th1 and Th17, leading to detrimental effects of neuroinflammation and demyelination. We previously reported that $3 H-1,2$-dithiole-3-thione (D3T), the structurally-simplest of the sulfurcontaining dithiolethiones, exerted a promising therapeutic effect in EAE. In the current study we report that 5-Amino-3-thioxo-3H-(1,2)dithiole-4-carboxylic acid ethyl ester (ACDT), a substituted derivative of D3T, exhibits anti-inflammatory properties in EAE. ACDT, administered post immunization, delayed disease onset and reduced disease severity in chronic C57BL/6 EAE, and ACDT, administered during disease remission, suppressed disease relapse in relapsing-remitting SJL/J EAE. Further analysis of the cellular and molecular mechanisms underlying the protective effects of ACDT in EAE revealed that ACDT inhibited pathogenic T cell infiltration, suppressed microglia activation, repressed neurotoxic A1 astrocyte generation, lessened blood-brain barrier disruption, and diminished MMP3/9 production in the CNS of EAE. In summary, we demonstrate that ACDT suppresses neuroinflammation and ameliorates disease severity in EAE through multiple cellular mechanisms. Our findings suggest the potential of developing ACDT as a novel therapeutic agent for the treatment of MS/EAE.

Keywords: MS/EAE; Microglia; Th1/Th17; A1 astrocyte; ACDT; Neuroinflammation; Bloodbrain barrier 


\section{Introduction}

Multiple sclerosis (MS) is an autoimmune disorder characterized by the CNS infiltration of myelin-specific pathogenic $\mathrm{T}$ cells and subsequent brain inflammation in association with myelin sheath deterioration (Fletcher et al., 2010; Kurschus, 2015; Yadav et al., 2015). Experimental autoimmune encephalomyelitis (EAE) is a commonly used animal model to study MS. Several T cell subsets, including $\mathrm{CD}^{+} \mathrm{T}$ cell lineages of $\mathrm{Th} 1$ and $\mathrm{Th} 17, \mathrm{CD} 8^{+} \mathrm{T}$ cells, and IL-17producting $\gamma \delta \mathrm{T}$ cells, are essential for the pathogenesis of EAE (Camara et al., 2013; Codarri et al., 2011; Grifka-Walk et al., 2015; Jager et al., 2009; McWilliams et al., 2015; Paul et al., 2015). In addition, antigen presenting cells such as peripheral dendritic cells and CNS microglial cells have been shown to play a pivotal role in the promotion of differentiation and reactivation of pathogenic T cells, respectively (Blink et al., 2009; Croxford et al., 2015).

In our previous study, we demonstrated the amelioration of EAE by the agent $3 \mathrm{H}$-1,2-dithiole-3thione (D3T) (Kuo et al., 2016). The promising in vivo activity of D3T, the structurally-simplest of the sulfur-containing dithiolethiones, is a composite of its physicochemical, pharmacokinetic, and pharmacodynamic properties. Further improvement in the activity of D3T can be made by optimizing these properties through structural modifications. As such, we aimed to determine the influence of modifications of D3T structure on EAE activity. We sought a molecule that retains the dithiolethione pharmacophore, but has functional groups that are easily modified. Our search identified compound 5-Amino-3-thioxo-3H-(1,2)dithiole-4-carboxylic acid ethyl ester (ACDT), a dithiolethione with ethyl ester and primary amine functional groups (Supplemental Fig. 1). We, therefore, decided to investigate the therapeutic efficacy of ACDT in EAE. 
In the present study, we report that ACDT exerts anti-inflammatory effects on the amelioration of neuroinflammation in EAE. Our results showed ACDT, administered post immunization, delayed disease onset and reduced disease severity in chronic C57BL/6 EAE, and ACDT, administered during disease remission, effectively suppressed disease relapse in relapsingremitting SJL/J EAE. Further investigation of cellular and molecular mechanisms underlying the protective effect of ACDT in EAE revealed that ACDT suppressed CNS infiltration of pathogenic T cells, including Th1 and Th17 cells. In addition, ACDT exhibited a suppressive effect on microglia (MG) activation in LPS-stimulated primary MG and in the CNS of EAE. Furthermore, through analyzing neuroinflammatory status in the CNS of vehicle- and ACDTtreated EAE mice, we found that several key inflammatory mediators related to EAE pathogenesis, including TNFa, osteopontin, ICAM-1, CD40, myeloperoxidase, CCL5, and CCL6, were suppressed by ACDT. The modulatory effect on the generation of A1 astrocytes, which have been shown to exert a neurotoxic effect (Liddelow et al., 2017), was also observed in the CNS of ACDT-treated EAE mice compared to that in vehicle-treated EAE controls. Importantly, ACDT treatment lessened blood-brain barrier (BBB) disruption, and that is associated with suppressed MMP3 and MMP9 production in the spinal cord of ACDT-treated EAE mice. In summary, we demonstrate that ACDT alleviates neuroinflammation in EAE through inhibiting pathogenic $\mathrm{T}$ cell infiltration, suppressing MG activation, and lessening BBB disruption in the CNS of EAE. Our findings suggest that ACDT could be developed as a potential therapeutic agent for the treatment of MS/EAE.

\section{Material and Methods}

\subsection{Animals}


C57BL/6 and SJL/J mice, purchased from The Jackson Laboratory (Bar Harbor, ME), were used in this study. Mice were bred with free accessed food and water, and housed in rooms with controlled temperature, humidity, and 12:12 hour light:dark cycle. All animal studies procedures were approved by the Purdue University Animal Care and Use Committee (PACUC).

\subsection{Reagents}

5-Amino-3-thioxo-3H-(1,2)dithiole-4-carboxylic acid ethyl ester (ACDT) was purchased from Oakwood Chemicals (West Columbia, SC). Complete Freund's adjuvant (CFA), Solutol ${ }^{\circledR}$ HS 15, Evans blue, collagenase, deoxyribonuclease I (DNase I), phorbol myristate acetate (PMA), ionomycin, paraformaldehyde, hematoxylin, eosin, cresyl violet, and luxol fast blue (LFB) were purchased from Sigma-Aldrich (St. Louis, MO). Antibodies for FACS analysis, including Alexa Fluor 488 anti-mouse CD4 (clone: RM4-5), APC anti-mouse IL-17A (clone: TC11-18H10.1), PE/Cy7 anti-mouse IFN $\gamma$ (clone: XMG1.2), APC anti-mouse CD45 (clone: 30-F11), PE antimouse CD11b (clone: M1/70), APC anti-mouse CD40 (clone: 3/23), PE anti-mouse CD80 (clone: 16-10A1), PE/Cy7 anti-mouse CD80 (clone: 16-10A1), PE anti-mouse CD86 (clone: GL1), and PE/Cy7 anti-mouse CD86 (clone: GL-1) were purchased from BioLegend (San Diego, CA). Antibodies for western blots, including anti-MMP9 (clone: L51/82) and MMP3 (clone: M4405F10) and reagents for intracellular staining, including Brefeldin A solution, fixation buffer, and permeabilization wash buffer, were purchased from BioLegend (San Diego, CA). Mycobacterium tuberculosis H37 RA and antibodies of $\beta$-actin (clone: C4/actin) and HRP goat anti-mouse IgG for western blots were purchased from BD (Sparks, MD). Pertussis toxin was purchased from List Biological Labs (Campbell, CA). Percoll was purchased from GE 
Healthcare Life Sciences (Pittsburgh, PA). Metaphosphoric acid was purchased from Alfa Aesar (Tewksbury, MA).

\subsection{EAE Induction}

C57BL/6 mice were immunized with $\mathrm{MOG}_{35-55}$ and SJL/J mice were immunized with PLP 139-151 $_{1}$ to induce chronic and relapsing-remitting EAE, respectively. Disease induction was conducted as previously described (Kuo et al., 2016). In brief, 7-9 weeks old female mice were subcutaneously injected with an emulsion of $200 \mu \mathrm{g} \mathrm{MOG}_{35-55}$ peptide (C57BL/6 model) or $100 \mu \mathrm{g} \mathrm{PLP}_{139-151}(\mathrm{SJL} / \mathrm{J}$ model) in CFA containing Mycobacterium tuberculosis $\mathrm{H} 37$ RA (final concentration $2 \mathrm{mg} / \mathrm{ml}$ ) on day 0, and followed by intraperitoneal (i.p.) administration of 200ng pertussis toxin in PBS on day 0 and day 2. EAE mice were then randomly grouped into two groups. One group was i.p administered with vehicle $(0.5 \mathrm{ml} 5 \%$ Solutol HS 15 / 0.9\% saline / DMSO) and the other group was administered with ACDT (0.5ml 5\% Solutol HS $15 / 0.9 \%$ saline / 20mg/kg ACDT dissolved in DMSO). The clinical scores of vehicle- and ACDT-treated EAE mice were evaluated based on the following criteria; 0: normal mouse, no overt signs of disease; 1: limp tail or hind limb weakness; 2: limp tail and hind limb weakness; 3: partial hind limb paralysis; 4: complete hind limb paralysis; and 5: moribund state. Animals with clinical scores of 5 were euthanized.

\subsection{Brain and Spinal Cord Tissue Processing}

EAE mice were anesthetized and perfused with ice-cold PBS. The brain and spinal cord were then harvested and homogenized. The brain tissues were homogenized with HBSS buffer only, and the spinal cord tissues were homogenized with HBSS buffer, containing collagenase and 
DNase I, followed by $37^{\circ} \mathrm{C}$ incubation for 45 minutes. Homogenized brain and spinal cord tissue samples were then filtered through $70-\mu \mathrm{m}$ nylon cell strainers. After centrifugation, cells prepared from brain and spinal cord tissues were subjected to mononuclear cell isolation or RNA extraction. In addition, cells prepared from spinal cord tissues were subjected to cytokine array and western blots.

\subsection{Isolation of Mononuclear Cells from Brain and Spinal Cord Tissues}

Mononuclear cell isolation was processed as previously described (Kuo et al., 2016). Cells prepared from brain and spinal cord tissues were then resuspended in $8 \mathrm{ml} 30 \%$ Percoll and underlayered with $4 \mathrm{ml} 70 \%$ Percoll followed by centrifugation for 25 minutes at $1000 \mathrm{~g}$ at room temperature (RT). The mononuclear cells were isolated from the interface between $30 \%$ and $70 \%$ Percoll.

\subsection{FACS Analysis for Intracellular Cytokines and Surface Markers}

Mononuclear cells isolated from the spinal cord and brain of C57BL/6 EAE animals were stimulated with PMA (50ng/ml) and ionomycin $(750 \mathrm{ng} / \mathrm{ml})$ in the presence of Brefeldin A solution $(1 \mu \mathrm{l} / \mathrm{ml})$. 5 hours later, cells were then fixed and permeabilized followed by intracellular staining with antibodies of Alexa Fluor 488 anti-mouse CD4 antibody, PE/Cy7 antimouse IFN $\gamma$, and APC anti-mouse IL-17. IFN $\gamma$ - and IL-17-expressing $\mathrm{CD}^{+} \mathrm{T}$ cells were then determined by FACS analysis. For MG surface marker analysis, the isolated mononuclear cells were stained with PE anti-mouse CD11b and APC anti-mouse CD45 with PE/Cy7 anti-mouse CD80 or PE/Cy7 anti-mouse CD86, and primary MG were stained with PE anti-mouse CD80, 
PE/Cy7 anti-mouse CD86, and APC anti-mouse CD40, followed by FACS analysis (BD FACSVerse).

\subsection{Cytokine Array}

The Proteome Profiler ${ }^{\mathrm{TM}}$ Array - Mouse XL Cytokine Array Kit (R\&D Systems, ARY028) was used to determine the relative expression level of cytokines and chemokines in the spinal cord of EAE mice. Experiments were conducted according to the manufacturer's instructions. Cells prepared from spinal cord tissues were lysed in RIPA buffer [50mM Tris-HCl (pH8.0), 150mM $\mathrm{NaCl}, 1 \% \mathrm{NP}-40,0.5 \%$ sodium deoxycholate, $0.3 \% \mathrm{SDS}, 1 \mathrm{mM}$ PMSF, and $1 \mathrm{X}$ protease inhibitor cocktail]. Protein concentration was detected by PierceTM BCA Protein Assay Kit (Thermo

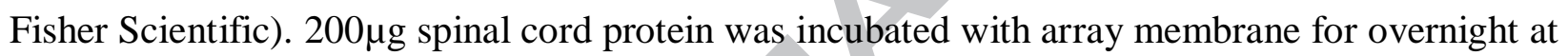
$4^{\circ} \mathrm{C}$ on a rocking platform shaker. After incubation, the membrane was washed and incubated with the Detection Antibody Cocktail for 1 hour at RT. The membrane was then washed and incubated with $1 \mathrm{X}$ Streptavidin-HRP for 30 minutes at RT followed by washing and incubation with Chemi Reagent Mix. Signal dots appeared on the membrane were then detected by X-ray film (FUJIFILM Super RX-N). The fold change of signal dots representing cytokine and chemokine expression was measured and determined by using protein array analyzer running under Image J (National Institutes of Health).

\subsection{Measurement of glutathione levels in the Brain and Spinal Cord of EAE}

Brain and spinal cord tissues collected from EAE mice were subjected to glutathione (GSH) assay. The total GSH level was measured by using Glutathione (GSSG/GSH) Detection Kit (Enzo Life Sciences, ADI-900-160). The brain and spinal cord tissues were homogenized in ice- 
cold 5\% (w/v) metaphosphoric acid (20ml/g tissue). Following centrifugation, supernatants were subjected to absorbance reading (405 nm) by BioTek Synergy HT microplate reader (Winooski, VT) to determine GSH levels according to manufacturer's instruction.

\subsection{Western Blot Analysis}

Cells prepared from spinal cord tissues were lysed in RIPA buffer [50mM Tris- $\mathrm{HCl}(\mathrm{pH} 8.0)$, $150 \mathrm{mM} \mathrm{NaCl}, 1 \% \mathrm{NP}-40,0.5 \%$ sodium deoxycholate, $0.3 \%$ SDS, 1mM PMSF, and 1X protease inhibitor cocktail]. Protein concentration was detected by Pierce ${ }^{\mathrm{TM}}$ BCA Protein Assay Kit (Thermo Fisher Scientific). Tissue Protein samples were separated on 10\% SDS-PAGE and then transferred to polyvinylidene difluoride (PVDF) membranes (Millipore). Blots were then incubated with MMP9, MMP3, or $\beta$-actin antibody. Protein signals were detected by using Immobilon $^{\mathrm{TM}}$ Western Chemiluminescent HRP Substrate (Millipore) and X-ray film. The quantification of protein signals was measured by using Image $\mathrm{J}$ software.

\subsection{Primary MG Cell Culture}

Primary MG were generated as previously described (Kuo et al., 2017). In brief, cerebral cortical cells from 1-2 days old neonatal mice were plated in $75-\mathrm{cm}^{2}$ culture flasks in Dulbecco Modified Eagle Medium/F12 (DMEM/F12) $\left(\right.$ HyClone $\left.^{\mathrm{TM}}\right)$ supplemented with 10\% heat-inactivated FBS, containing $2 \mathrm{mM}$ glutamine and 1X antibiotic/antimycotic (complete medium). On day 3 and 6 after plating, medium was removed and replenished with fresh complete medium containing 10 ng/ml GM-CSF. MG were harvested at Day 13 or 14 by shaking the flasks at 350 rpm for 30 minutes at $37^{\circ} \mathrm{C}$. Harvested cells were then seeded in the tissue culture wells. After overnight resting, cells were stimulated with LPS for $24 \mathrm{~h}$ to induce surface expression of CD40, CD80, and 
CD86, or for $1.5 \mathrm{~h}$ and $3 \mathrm{~h}$ to induce inflammatory gene expression based on our previous study (Kuo et al., 2017).

\subsection{Real-time RT-PCR}

Primary MG and cells prepared from brain and spinal cord tissues were subjected to RNA extraction followed by cDNA synthesis. Expression of $I l-1 \beta, I l-12 p 35, I l-12 p 40, I l-23 p 19, I l-6$, Gm-csf, Il-1 $\alpha$, Tnf $\alpha$, Gatal, H2-D1, and Serpingl were detected by real-time RT-PCR as previous described (Yen et al., 2008). The primers used were $I l-1 \beta$ : sense 5'CCCTGCAGCTGGAGAGTGTGGA-3' and antisense 5'-TGTGCTCT GCTTGGAGGTGCTG3'; Il-12p35: sense 5'-CTGTGCCTTGGTAGCATCTATG $\quad-3^{\prime}$ ' and anti-sense 5'GCAGAGTCTCGCCATTATGATTC-3'; Il-12p40: $\quad$ sense $\quad$ 5'-TGGTTTGCCATCGTTT TGCTG-3' and antisense 5'-ACAGGTGAGGTTCACTGTTTCT-3'; Il-23p19: sense 5'-TGCTG GATTGCAGAGCAGTAA-3' and anti-sense 5'-GCATGCAGAGATTCCGAGAGA-3'; Il-6: 5'-TCСTCTCTGCAAGAGACTTCCATCC-3' ${ }^{\prime}$ and 5' GGGAAGGCCGTGGTTGTCA CC-3'; Gm-csf: sense 5'-ATGCCTGTCACGTTGAATGAAG-

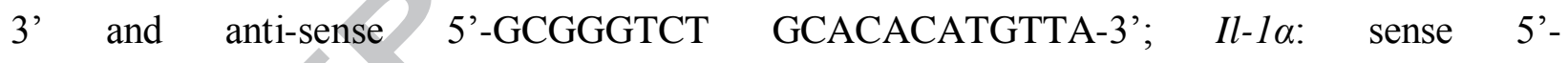
CGCTTGAGTCGGCAAAGAAAT-3' and antisense 5'- CTTCCCGTTGCTTGACGTTG-3'; Tnfo: sense 5'-ATGGCCTCCCTCTCATCAGT-3' and antisense 5'CTTGGTGGTTTGCTACGACG-3'; Gatal: sense 5'- GTGAACAGCATGAGGGG TTT-3' and antisense 5'- GTTTTGTTGCCTCTGGGTGT-3'; H2-D1: sense 5'-TCCGAGATTGT AAAGCGTGAAGA-3' and anti-sense 5'- ACAGGGCAGTGCAGGGATAG-3'; Serping1 sense $\quad 5^{\prime}-\quad$ ACAGCCCCCTCTGAATTCTT-3' and antisense 5'GGATGCTCTCCAAGTTGCTC-3’' 


\subsection{Evans Blue BBB Permeability Assay}

Evans blue extravasation of CNS tissues of EAE was determined as previously described with modifications (Fairless et al., 2012; Lee et al., 2012). Mice were intravenously (i.v.) administrated $4 \mathrm{ml} / \mathrm{kg} 2 \%(\mathrm{w} / \mathrm{v})$ Evans blue dye. After 2 hours circulation, mice were anesthetized and perfused with PBS. The brain and spinal cord of EAE mice were then carefully dissected to assess Evans blue leakage.

\subsection{Histopathology}

EAE mice were sacrificed and perfused with PBS. The spinal cord was then removed from spinal columns, and the lumbar region of spinal cord was then separated and fixed in $4 \%$ paraformaldehyde at $4^{\circ} \mathrm{C}$ for overnight. Fixed tissues were embedded in paraffin for sectioning. Serial $8 \mu \mathrm{m}$ sections were cut on a microtome (Leica RM2155). Cross sections were used for hematoxylin and eosin $(\mathrm{H} \& \mathrm{E})$ or $\mathrm{LFB} / \mathrm{cresyl}$ violet staining. Stained spinal cord slides were examined and imaged under magnification (microscope: BX53, Olympus; camera: EXi Aqua, Q Imaging). Image $J$ was used to quantify the area of cell infiltration and the percentage of demyelination by an experimenter blinded to experimental groups.

\subsection{Statistical Analysis}

Experimental results are given as mean \pm SEM. Comparisons between groups were done by using the Mann-Whitney $U$ test, unpaired $t$ test, or one-way ANOVA test. Statistical significance was determined as $p$ values $\leq 0.05$. All statistical analysis was performed by using GraphPad Prism 5 software (La Jolla, CA). 


\section{Results}

\subsection{ACDT ameliorates disease severity in chronic and relapsing-remitting EAE}

To determine whether ACDT exerts a therapeutic potential in EAE, C57BL/6 mice immunized with $\mathrm{MOG}_{35-55}$ were treated with ACDT every day starting from day 1 post immunization, and the clinical score of vehicle- and ACDT-treated EAE mice was followed for a period of 30 days. Our results showed that ACDT treatment not only delayed disease onset but also reduced disease severity in EAE, with the maximum disease score of $2.2 \pm 0.3$ compared to that of $4.1 \pm 0.2$ in vehicle-treated EAE controls (Fig. 1A and Table). When comparing the cumulative score, ACDT-treated EAE mice had a much lower cumulative disease score of $26.6 \pm 4.6$ compared to that of $64.3 \pm 2.8$ in vehicle-treated EAE controls (Fig. 1A Table).

We then explored the therapeutic potential of ACDT on the suppression of disease relapse in EAE. Relapsing-remitting EAE was induced in SJL/J mice, and EAE mice were administered with vehicle or ACDT during disease remission (disease score below 1.5). ACDT treatment suppressed disease relapse in EAE, with the maximum disease score of post treatment only reaching $1.4 \pm 0.2$, whereas vehicle-treated EAE controls developed disease relapse following remission, with the maximum disease score of post treatment reaching $3.0 \pm 0.3$ (Fig. 1B and Table). When comparing the cumulative score of post treatment, ACDT-treated EAE mice had a much lower cumulative score of $12.6 \pm 1.9$ compared to vehicle-treated EAE mice of $30.2 \pm 2.7$ (Fig. 1B Table). Taken altogether, these results demonstrate that ACDT confers protection against EAE through ameliorating disease severity and suppressing disease relapse in EAE.

3.2 ACDT suppresses CNS infiltration of encephalitogenic Th1 and Th17 cells in EAE 
The CNS infiltration of encephalitogenic Th1 and Th17 cells plays a pivotal role in the pathogenesis of EAE (Jager et al., 2009; McWilliams et al., 2015). To investigate whether ACDT suppresses encephalitogenic $\mathrm{CD}^{+} \mathrm{T}$ cell infiltration of $\mathrm{CNS}$, mononuclear cells were isolated from the brain and spinal cord of vehicle- and ACDT-treated EAE mice at day 13-14 post immunization, and the isolated cells were then subjected to FACS analysis of total CD $4^{+} \mathrm{T}$ cells and $\mathrm{CD}^{+} \mathrm{T}$ cells expressing IFN $\gamma$ (Th1) and IL-17 (Th17). We observed a significant increase of infiltrating $\mathrm{CD} 4^{+} \mathrm{T}$ cells in the brain and spinal cord of vehicle-treated EAE mice (Fig. 2A), and those $\mathrm{CD}^{+} \mathrm{T}$ cells positively expressed IFN $\gamma$ (Fig. 2B) or IL-17 (Fig. 2C). In contrast, the number of infiltrating $\mathrm{CD}^{+} \mathrm{T}$ cells was largely reduced in the CNS of ACDTtreated EAE mice compared to that in vehicle-treated EAE controls, and that is associated with decreased infiltration of encephalitogenic Th1 and Th17 cells (Fig. 2). Collectively, these results suggest that ACDT-conferred protection in EAE might be mediated through the suppression of CNS infiltration of encephalitogenic Th1 and Th17 cells.

\subsection{ACDT suppresses $M G$ activation}

MG are the major CNS resident immune cells and function as antigen presenting cells to reactivate pathogenic $\mathrm{T}$ cells in lesion sites, thereby exacerbating the disease by epitope spreading (McMahon et al., 2005; Tompkins et al., 2002). MG activation leads to increased secretion of diverse cytokines and enhanced expression of surface maturation markers CD40, CD80, and CD86. To investigate whether ACDT exerts a suppressive effect on MG activation, we cultured primary MG and activated MG with LPS in the presence or absence of ACDT. LPS upregulated surface expression of CD40, CD80, and CD86, whereas ACDT suppressed LPSinduced CD40, CD80, and CD86 upregulation (Fig. 3A). Moreover, LPS upregulated 
inflammatory cytokine expression, including IL-23p19, IL-1 $\beta$, and IL-6 required for Th17 differentiation and reactivation, and IL-12p35 and IL-12p40 required for Th1 differentiation and reactivation. In contrast, ACDT suppressed LPS-induced IL-12p35, IL-12p40, IL-1 $\beta$, IL-6, and IL-23p19 expression (Fig. 3B). GM-CSF, which was shown to promote neuroinflammation in EAE (El-Behi et al., 2011; Shiomi et al., 2015), was also upregulated in MG activated with LPS, but downregulated in MG activated with LPS in the presence of ACDT (Fig 3B).

To further investigate whether ACDT modulates MG activation in vivo, EAE was induced and mononuclear cells were isolated from the spinal cord and brain of vehicle- and ACDT-treated EAE mice at day 13-14 post immunization. The isolated cells were then subjected to CD45, CD11b, CD80, and CD86 staining followed by FACS analysis. MG were determined based on their positive expression of $\mathrm{CD} 11 \mathrm{~b}$ and intermediated expression of CD45. Although there was no significant difference of CD86 expression on the surface of $\mathrm{CD} 45^{\mathrm{int}} \mathrm{CD} 11 \mathrm{~b}^{+} \mathrm{MG}$ isolated from the spinal cord and brain of vehicle- and ACDT-treated EAE mice, CD80 expression was significantly reduced on the surface of $\mathrm{CD} 45^{\mathrm{int}} \mathrm{CD} 11 \mathrm{~b}^{+} \mathrm{MG}$ isolated from the spinal cord and brain of ACDT-treated EAE mice compared to that in vehicle-treated EAE controls (Fig. 3C). Altogether, these results suggest that ACDT possesses a suppressive effect on MG activation in vitro as well as partially in vivo.

\subsection{ACDT lessens neuroinflammation in EAE}

To further investigate whether ACDT-mediated suppression of pathogenic $\mathrm{T}$ cell infiltration and MG activation could lead to the alleviation of neuroinflammation in EAE, the spinal cord harvested from vehicle- and ACDT-treated EAE mice was subjected to cytokine array to determine the expression of inflammatory mediators. We found that chemokines, related to the 
recruitment of inflammatory immune cells, including CCL5, CCL6, CCL17, and CXCL10, were expressed in the spinal cord of EAE mice, but their expression was suppressed in the spinal cord of ACDT-treated EAE mice (Fig. 4A). In addition, inflammatory mediators that are associated with pathogenesis of EAE, such as osteopontin, CD40, ICAM-1, and myeloperoxidase, were also expressed in the spinal cord of EAE, but suppressed in the spinal cord of ACDT-treated EAE mice (Fig. 4A).

A recent study shows that IL- $1 \alpha, \mathrm{TNF} \alpha$, and C1q, produced by activated $\mathrm{MG}$, induce neurotoxic reactive astrocytes that are named "A1 astrocytes". Importantly, these neurotoxic A1 astrocytes are also present in post-mortem brain tissues of MS patients (Liddelow et al., 2017). In our array results, we found that IL- $1 \alpha$ was expressed in the spinal cord of vehicle-treated EAE controls, but suppressed in the spinal cord of ACDT-treated EAE mice. We speculated that ACDT might regulate neurotoxic A1 astrocytes in the CNS of EAE through modulating the expression of inflammatory molecules required for A1 astrocyte generation. We then measured IL-1 $\alpha$, TNF $\alpha$, and C1q expression in the CNS of vehicle- and ACDT-treated EAE mice. Although we did not detect the expression of $\mathrm{Clq}$ in the CNS of EAE mice (data not shown), the expression of TNF $\alpha$ and IL-1 $\alpha$ was largely suppressed in the spinal cord and brain of ACDT-treated EAE mice compared to those in vehicle-treated EAE controls (Fig 4B). Subsequently, we determined the levels of A1 astrocytes in the spinal cord and brain of vehicle- and ACDT-treated EAE mice by measuring A1 astrocyte specific genes, including Ggta1, H2-D1, and Serping1. The expression of Ggta1, H2-D1, and Serping1 was suppressed in the CNS of ACDT-treated EAE mice compared to that in vehicle-treated EAE controls (Fig. 4C). Taken altogether, these results demonstrate that ACDT lessens neuroinflammation and potentially suppresses neurotoxic A1 astrocyte generation in EAE. 


\subsection{ACDT lessens BBB disruption and alleviates spinal cord pathology in EAE}

To determine whether ACDT lessens EAE-induced BBB disruption, during the peak of disease vehicle- and ACDT-treated EAE mice were subjected to Evans Blue injection followed by the brain and spinal cord tissue isolation to assess BBB integrity. Evans blue leakage was clearly observed in the CNS especially in the spinal cord of vehicle-treated EAE controls, indicating a compromised BBB integrity. On the other hand, the leakage of Evans blue was significantly reduced in the spinal cord and brain of ACDT-treated EAE mice, suggesting a well preserved BBB integrity in ACDT-treated EAE mice (Fig. 5A). Both MMP3 and MMP9 play a detrimental role in BBB disruption and breakdown in several neurodegenerative diseases (Fanjul-Fernandez et al., 2010; Gerwien et al., 2016; Kim et al., 2011; Konnecke et al., 2013), we thought to determine whether the beneficial effect of ACDT on BBB integrity in EAE is mediated through its regulatory effect on MMP3 and MMP9 production. We measured MMP3 and MMP9 expression in the spinal cord of vehicle- and ACDT-treated EAE mice, and found MMP3 and MMP9 were highly expressed in the spinal cord of vehicle-treated EAE controls. Conversely, the expression of MMP3 and MMP9 was largely suppressed in the spinal cord of ACDT-treated EAE mice (Fig 5B).

We further examined the histopathological features of spinal cord in vehicle- and ACDT-treated EAE mice. The spinal cord harvested from EAE mice was subjected to H\&E and LFB/cresyl violet staining to assess cell infiltration and demyelination, respectively. We observed a significant increase of infiltrating inflammatory cells and a moderate level of demyelination in the spinal cord of vehicle-treated EAE controls. In contrast, the histopathology of cell infiltration and demyelination was alleviated in the spinal cord of ACDT-treated EAE mice (Fig. 5C and D). 
Altogether, these results demonstrate that ACDT lessens BBB disruption, suppresses MMP3 and MMP9 production, inhibits inflammatory cell infiltration, and alleviates demyelination in the spinal cord of EAE.

\subsection{ACDT enhances GSH levels in the CNS of EAE}

The inflammatory process in EAE is associated with increased production of reactive oxygen species and reduced production of redox active compounds, such as GSH, in the CNS (Choi et al., 2015; Ljubisavljevic et al., 2012; Mohamed et al., 2003; Morales Pantoja et al., 2016; Ruuls et al., 1995). Our group has previously demonstrated that ACDT strongly induced the production of GSH in SH-SY5Y cells (Brown et al., 2016). To assess whether ACDT induces GSH production in the CNS to modulate neuroinflammation in EAE, the spinal cord and brain were harvested from vehicle- and ACDT-treated EAE mice and subjected to GSH analysis. We found that GSH levels were higher in the brain and spinal cord of ACDT-treated EAE mice than those in vehicle-treated EAE controls (Fig. 6), suggesting that the induction of GSH by ACDT might contribute to its suppressive effect on neuroinflammation in EAE.

\section{Discussion}

Dithiolethiones have well-demonstrated protective effects in a number of neurodegenerative and neurological diseases, including Alzheimer disease, UV-induced retinal pigment epithelium cell injury, stroke, and EAE (Cui et al., 2017; Kuo et al., 2016; Kuo et al., 2017; Li et al., 2016; Wang et al., 2017). Our group previously reported the anti-inflammatory properties of D3T, the structurally simplest of the dithiolethiones, in EAE (Kuo et al., 2016). As a continuation of our study of D3T, we sought to assess the effect of modifying the dithiolethione core structure on 
EAE activity. ACDT structurally retains the dithiolethione pharmacophore, but has two versatile functional groups (carboxylic acid ethyl ester and primary amine) that are readily amendable for future modifications. As the therapeutic effect of ACDT in EAE is unknown, we therefore investigated its activity in two well-established EAE models, chronic C57BL/6 EAE and relapsing-remitting SJL/J EAE. We observed that in the chronic EAE, ACDT treatment delayed disease onset for an average of 4 days compared to controls, minimized maximum disease score to the average of 2.2 as opposed to 4.1 for controls, and ameliorated disease severity with cumulative score of 26.6 compared to 64.3 for controls. More importantly, in the relapsingremitting EAE, ACDT, administered during disease remission, prevented disease relapse, and the cumulative score of post treatment significantly reduced to 12.6 compared to 30.2 for controls. Based on these findings, we concluded that ACDT exerts a therapeutic effect in chronic and relapsing-remitting EAE by ameliorating disease severity and preventing disease progression.

We further investigated the cellular mechanisms underlying the protective effect of ACDT in EAE. The infiltration of pathogenic Th1 and Th17 cells has been shown to play a critical role in the pathogenesis of EAE (El-Behi et al., 2011; Jager et al., 2009). ACDT treatment suppressed the infiltration of Th1 and Th17 cells in the spinal cord and brain of EAE mice compared to those in vehicle-treated EAE controls. In addition, our observation of reduced total infiltrating $\mathrm{CD}^{+} \mathrm{T}$ cells in the CNS of ACDT-treated EAE mice led us to speculate that ACDT might lessen disease-induced BBB disruption. Indeed, we found that EAE mice treated with vehicle had a compromised BBB integrity and EAE mice treated with ACDT exhibited a well preserved BBB integrity, as the profound leakage of Evans blue was only observed in the spinal cord of vehicle-treated EAE controls but not in the spinal cord of ACDT-treated EAE mice. Further analysis of the spinal cord of EAE revealed that metalloproteinases MMP3 and MMP9, essential 
proteases contributing to the cleavage of $\mathrm{BBB}$ basement membrane and degradation of tight junction proteins (Lakhan et al., 2013; Yang et al., 2007), were largely expressed in the spinal cord of vehicle-treated EAE controls but dramatically suppressed in the spinal cord of ACDTtreated EAE mice. These results correlate with the histopathology of spinal cord in which increased cell infiltration and enhanced demyelination were observed in vehicle-treated EAE controls but not in ACDT-treated EAE mice. CNS resident cells, including MG and brain endothelial cells, and infiltrating inflammatory immune cells are the cell populations, producing MMP3 and MMP9 under neuroinflammatory conditions (Amtul et al., 2014; Brkic et al., 2015; Yang et al., 2007). Further investigation would be required to dissect which cell types that secret MMP3 and MMP9 following EAE are modulated by the treatment of ACDT.

A recent study shows that activated microglia induce neurotoxic reactive astrocytes in neurodegenerative diseases (Liddelow et al., 2017). Cytokines TNF $\alpha$ and IL-1 $\alpha$ and complement component $\mathrm{C} 1 \mathrm{q}$ released by activated microglia promote the generation of reactive astrocytes that exert a toxic effect on neurons. These reactive astrocytes were subsequently named as "A1 astrocytes". A1 astrocytes are rapidly generated in vivo following CNS injury and present in many human neurodegenerative diseases, including Huntington's disease, Alzheimer's disease, ALS, Parkinson's disease, and MS (Liddelow et al., 2017). A1 astrocytes express a spectrum of signature genes that can be used for screening or detecting these neurotoxic reactive astrocytes in the CNS of neurodegenerative diseases. In the present study, we examined whether ACDTmediated amelioration of neuroinflammation could lead to reduced A1 astrocyte generation in EAE. We observed ACDT inhibited a spectrum of cytokines, chemokines, and inflammatory mediators in the CNS of EAE. Importantly, essential cytokines required for the generation of neurotoxic A1 astrocytes, including TNF $\alpha$ and IL-1 $\alpha$, were suppressed in the CNS of ACDT- 
treated EAE mice, although we did not detect the expression of C1q in the CNS of EAE. Subsequent analysis of A1 astrocytes in the CNS of EAE revealed that ACDT suppressed the expression of A1 astrocyte signature genes, including Ggta1, H2-d1, and Serping1. Thus, our results demonstrated that ACDT ameliorated neuroinflammation and that might subsequently reduce neurotoxic A1 astrocyte generation in EAE. However, a comprehensive study through measuring additional A1markers is required to further confirm the suppressive effect of ACDT on the generation of $\mathrm{A} 1$ astrocytes in the CNS of EAE. Moreover, whether the reduction of A1 astrocyte generation by ACDT could lead to decreased neuronal injury in EAE would require further investigation to reveal potential neuroprotective effects of ACDT in EAE.

Our previous study demonstrated that D3T, the structurally-simplest dithiolethione, ameliorated disease of EAE (Kuo et al., 2016). In the current study, we showed ACDT, a dithiolethione with ethyl ester and primary amine functional groups, alleviated disease severity to a similar degree as D3T in EAE. These results suggest a potential use of dithiolethiones as novel therapies for EAE/MS. As dithiolethiones are known to potently activate Nrf2 (Jia et al., 2008; Tran et al., 2009), the protective effect of D3T and ACDT in EAE might be mediated through Nrf2 defense pathway. Currently, there is only one FDA-approved Nrf2 activator on the market, dimethyl fumarate (DMF) (Alroughani et al., 2017). Based on the success of this drug in the clinic, the development of further Nrf2 activators is warranted. Additionally, DMF activates Nrf2 via the depletion of GSH, which generates oxidative stress (Lehmann et al., 2007; Xie et al., 2015). This has the potential effect of inducing cellular injury. In contrast, dithiolethiones activate Nrf2 in a process that does not trigger oxidative stress onto the cell (Brown et al., 2014; Giustarini et al., 2014; Kwak et al., 2003). Thus, dithiolethiones, such as D3T and ACDT, may represent better 
therapeutic agents for the treatment of EAE/MS through direct activation of the Nrf2 pathway without compromising the beneficial effect of GSH.

\section{Conclusions}

We reported for the first time that ACDT conferred protection against the autoimmune inflammatory disease EAE. We showed ACDT inhibited CNS infiltration of pathogenic $\mathrm{CD}^{+} \mathrm{T}$ cells, including Th1 and Th17 cells, and ACDT suppressed MG activation through inhibiting the expression of surface maturation markers and the production of inflammatory cytokines. In addition, ACDT lessened BBB disruption in the spinal cord of EAE through inhibiting MMP3 and MMP9 production that resulted in a better preserved BBB integrity with decreased CNS infiltration of inflammatory immune cells and alleviated demyelination in the spinal cord of EAE. In summary, our study demonstrated that ACDT exerts a therapeutic potential for the treatment of MS/EAE by targeting several pathological pathways related to the development and progression of EAE.

\section{Acknowledgements}

This work was supported by grants from Anna Yoder MS fund to J-H. Yen and from Manchester University College of Pharmacy, Natural, and Health Sciences to D. A Brown.

\section{References}

Alroughani R, Ahmed SF, Behbehani R, Al-Hashel J (2017). Effectiveness and Safety of Dimethyl Fumarate Treatment in Relapsing Multiple Sclerosis Patients: Real-World Evidence. Neurology and therapy 6(2): 189-196.

Amtul Z, Hepburn JD (2014). Protein markers of cerebrovascular disruption of neurovascular unit: immunohistochemical and imaging approaches. Rev Neurosci 25(4): 481-507. 
Blink SE, Miller SD (2009). The contribution of gammadelta T cells to the pathogenesis of EAE and MS. Curr Mol Med 9(1): 15-22.

Brkic M, Balusu S, Libert C, Vandenbroucke RE (2015). Friends or Foes: Matrix Metalloproteinases and Their Multifaceted Roles in Neurodegenerative Diseases. Mediators Inflamm 2015: 620581.

Brown DA, Betharia S, Yen JH, Kuo PC, Mistry H (2016). Further structure-activity relationships study of substituted dithiolethiones as glutathione-inducing neuroprotective agents. Chem Cent J 10: 64 .

Brown DA, Betharia S, Yen JH, Tran Q, Mistry H, Smith K (2014). Synthesis and structure-activity relationships study of dithiolethiones as inducers of glutathione in the SH-SY5Y neuroblastoma cell line. Bioorganic \& medicinal chemistry letters 24(24): 5829-5831.

Camara M, Beyersdorf N, Fischer HJ, Herold MJ, Ip CW, van den Brandt J, et al. (2013). CD8(+) T cell help is required for efficient induction of EAE in Lewis rats. J Neuroimmuno/ 260(1-2): 17-27.

Choi BY, Kim JH, Kho AR, Kim IY, Lee SH, Lee BE, et al. (2015). Inhibition of NADPH oxidase activation reduces EAE-induced white matter damage in mice. Journal of neuroinflammation 12: 104.

Codarri L, Gyulveszi G, Tosevski V, Hesske L, Fontana A, Magnenat L, et al. (2011). RORgammat drives production of the cytokine GM-CSF in helper T cells, which is essential for the effector phase of autoimmune neuroinflammation. Nat Immunol 12(6): 560-567.

Croxford AL, Spath S, Becher B (2015). GM-CSF in Neuroinflammation: Licensing Myeloid Cells for Tissue Damage. Trends Immunol 36(10): 651-662.

Cui Y, Ma S, Zhang C, Li D, Yang B, Lv P, et al. (2017). Pharmacological activation of the Nrf2 pathway by $3 \mathrm{H}-1,2$-dithiole-3-thione is neuroprotective in a mouse model of Alzheimer disease. Behav Brain Res 336: 219-226.

El-Behi M, Ciric B, Dai H, Yan Y, Cullimore M, Safavi F, et al. (2011). The encephalitogenicity of T(H)17 cells is dependent on IL-1- and IL-23-induced production of the cytokine GM-CSF. Nat Immunol 12(6): 568-575.

Fairless R, Williams SK, Hoffmann DB, Stojic A, Hochmeister S, Schmitz F, et al. (2012). Preclinical retinal neurodegeneration in a model of multiple sclerosis: The Journal of Neuroscience 2012; 32(16):55855597. Annals of neurosciences 19(3): 121-122.

Fanjul-Fernandez M, Folgueras AR, Cabrera S, Lopez-Otin C (2010). Matrix metalloproteinases: evolution, gene regulation and functional analysis in mouse models. Biochim Biophys Acta 1803(1): 3-19. 
Fletcher JM, Lalor SJ, Sweeney CM, Tubridy N, Mills KH (2010). T cells in multiple sclerosis and experimental autoimmune encephalomyelitis. Clin Exp Immunol 162(1): 1-11.

Gerwien H, Hermann S, Zhang X, Korpos E, Song J, Kopka K, et al. (2016). Imaging matrix metalloproteinase activity in multiple sclerosis as a specific marker of leukocyte penetration of the blood-brain barrier. Sci Transl Med 8(364): 364ra152.

Giustarini D, Fanti P, Sparatore A, Matteucci E, Rossi R (2014). Anethole dithiolethione lowers the homocysteine and raises the glutathione levels in solid tissues and plasma of rats: a novel non-vitamin homocysteine-lowering agent. Biochemical pharmacology 89(2): 246-254.

Grifka-Walk HM, Giles DA, Segal BM (2015). IL-12-polarized Th1 cells produce GM-CSF and induce EAE independent of IL-23. European journal of immunology 45(10): 2780-2786.

Jager A, Dardalhon V, Sobel RA, Bettelli E, Kuchroo VK (2009). Th1, Th17, and Th9 effector cells induce experimental autoimmune encephalomyelitis with different pathological phenotypes. J Immunol 183(11): 7169-7177.

Jia Z, Zhu H, Trush MA, Misra HP, Li Y (2008). Generation of superoxide from reaction of 3H-1,2-dithiole3-thione with thiols: implications for dithiolethione chemoprotection. Molecular and cellular biochemistry 307(1-2): 185-191.

Kim EM, Hwang O (2011). Role of matrix metalloproteinase-3 in neurodegeneration. J Neurochem 116(1): 22-32.

Konnecke H, Bechmann 1 (2013). The role of microglia and matrix metalloproteinases involvement in neuroinflammation and gliomas. Clin Dev Immunol 2013: 914104.

Kuo PC, Brown DA, Scofield BA, Yu IC, Chang FL, Wang PY, et al. (2016). 3H-1,2-dithiole-3-thione as a novel therapeutic agent for the treatment of experimental autoimmune encephalomyelitis. Brain Behav Immun 57: 173-186.

Kuo PC, Yu IC, Scofield BA, Brown DA, Curfman ET, Paraiso HC, et al. (2017). 3H-1,2-Dithiole-3-thione as a novel therapeutic agent for the treatment of ischemic stroke through Nrf2 defense pathway. Brain Behav Immun 62: 180-192.

Kurschus FC (2015). T cell mediated pathogenesis in EAE: Molecular mechanisms. Biomed J 38(3): 183193. 
Kwak MK, Wakabayashi N, Itoh K, Motohashi H, Yamamoto M, Kensler TW (2003). Modulation of gene expression by cancer chemopreventive dithiolethiones through the Keap1-Nrf2 pathway. Identification of novel gene clusters for cell survival. The Journal of biological chemistry 278(10): 8135-8145.

Lakhan SE, Kirchgessner A, Tepper D, Leonard A (2013). Matrix metalloproteinases and blood-brain barrier disruption in acute ischemic stroke. Front Neurol 4: 32.

Lee E, Chanamara S, Pleasure D, Soulika AM (2012). IFN-gamma signaling in the central nervous system controls the course of experimental autoimmune encephalomyelitis independently of the localization and composition of inflammatory foci. Journal of neuroinflammation 9: 7.

Lehmann JC, Listopad JJ, Rentzsch CU, Igney FH, von Bonin A, Hennekes HH, et al. (2007). Dimethylfumarate induces immunosuppression via glutathione depletion and subsequent induction of heme oxygenase 1. The Journal of investigative dermatology 127(4): 835-845.

Li KR, Yang SQ, Gong YQ, Yang H, Li XM, Zhao YX, et al. (2016). 3H-1,2-dithiole-3-thione protects retinal pigment epithelium cells against Ultra-violet radiation via activation of Akt-mTORC1-dependent Nrf2HO-1 signaling. Sci Rep 6: 25525.

Liddelow SA, Guttenplan KA, Clarke LE, Bennett FC, Bohlen CJ, Schirmer L, et al. (2017). Neurotoxic reactive astrocytes are induced by activated microglia. Nature 541(7638): 481-487.

Ljubisavljevic S, Stojanovic I, Pavlovic R, Stojnev S, Stevanovic I, Sokolovic D, et al. (2012). The reduced glutathione and S-nitrosothiols levels in acute phase of experimental demyelination--pathophysiological approach and possible clinical relevancy. Neuroscience 219: 175-182.

McMahon EJ, Bailey SL, Castenada CV, Waldner H, Miller SD (2005). Epitope spreading initiates in the CNS in two mouse models of multiple sclerosis. Nat Med 11(3): 335-339.

McWilliams IL, Rajbhandari R, Nozell S, Benveniste E, Harrington LE (2015). STAT4 controls GM-CSF production by both Th1 and Th17 cells during EAE. Journal of neuroinflammation 12: 128.

Mohamed A, Shoker A, Bendjelloul F, Mare A, Alzrigh M, Benghuzzi H, et al. (2003). Improvement of experimental allergic encephalomyelitis (EAE) by thymoquinone; an oxidative stress inhibitor. Biomed Sci Instrum 39: 440-445.

Morales Pantoja IE, Hu CL, Perrone-Bizzozero NI, Zheng J, Bizzozero OA (2016). Nrf2-dysregulation correlates with reduced synthesis and low glutathione levels in experimental autoimmune encephalomyelitis. J Neurochem 139(4): 640-650. 
Paul S, Shilpi, Lal G (2015). Role of gamma-delta (gammadelta) T cells in autoimmunity. J Leukoc Biol 97(2): 259-271.

Ruuls SR, Bauer J, Sontrop K, Huitinga I, t Hart BA, Dijkstra CD (1995). Reactive oxygen species are involved in the pathogenesis of experimental allergic encephalomyelitis in Lewis rats. $J$ Neuroimmunol 56(2): 207-217.

Shiomi A, Usui T (2015). Pivotal roles of GM-CSF in autoimmunity and inflammation. Mediators Inflamm 2015: 568543.

Tompkins SM, Padilla J, Dal Canto MC, Ting JP, Van Kaer L, Miller SD (2002). De novo central nervous system processing of myelin antigen is required for the initiation of experimental autoimmune encephalomyelitis. J Immunol 168(8): 4173-4183.

Tran QT, Xu L, Phan V, Goodwin SB, Rahman M, Jin VX, et al. (2009). Chemical genomics of cancer chemopreventive dithiolethiones. Carcinogenesis 30(3): 480-486.

Wang L, Wang M, Hu J, Shen W, Yao Y, Wang X, et al. (2017). Protective effect of 3H-1, 2-dithiole-3thione on cellular model of Alzheimer's disease involves Nrf2/ARE signaling pathway. Eur J Pharmacol 795: 115-123.

Xie X, Zhao Y, Ma CY, Xu XM, Zhang YQ, Wang CG, et al. (2015). Dimethyl fumarate induces necroptosis in colon cancer cells through GSH depletion/ROS increase/MAPKs activation pathway. British journal of pharmacology 172(15): 3929-3943.

Yadav SK, Mindur JE, Ito K, Dhib-Jalbut S (2015). Advances in the immunopathogenesis of multiple sclerosis. Curr Opin Neurol 28(3): 206-219.

Yang Y, Estrada EY, Thompson JF, Liu W, Rosenberg GA (2007). Matrix metalloproteinase-mediated disruption of tight junction proteins in cerebral vessels is reversed by synthetic matrix metalloproteinase inhibitor in focal ischemia in rat. J Cereb Blood Flow Metab 27(4): 697-709.

Yen JH, Khayrullina T, Ganea D (2008). PGE2-induced metalloproteinase-9 is essential for dendritic cell migration. Blood 111(1): 260-270.

\section{Figure Legends}

Fig. 1 ACDT ameliorates disease severity in chronic and relapsing-remitting EAE 
(A) C57BL/6 mice ( $\mathrm{n}=18 /$ group) immunized with $\mathrm{MOG}_{35-55}$ were administered i.p. with vehicle or ACDT $(20 \mathrm{mg} / \mathrm{kg})$ every day starting from day 1 post immunization. (B) SJL/J mice ( $\mathrm{n}=9$ /group) were immunized with PLP ${ }_{139-151}$ to induce EAE. Following the first remission and before the onset of second relapse, EAE animals were administered i.p. with vehicle or ACDT $(20 \mathrm{mg} / \mathrm{kg})$ every day. The clinical score of EAE animals was followed, and EAE mortality rate, onset of disease, maximum score, and cumulative score were assessed. Data represent mean \pm SEM. Statistical significance was determined as: ${ }^{*} p<0.05$, $* * p<0.01$, and $* * * p<0.001$ by Mann-Whitney $U$ test.

\section{Fig. 2 ACDT suppresses CNS infiltration of encephalitogenic Th1 and Th17 cells in EAE}

C57BL/6 mice ( $\mathrm{n}=9 /$ group) were subjected to EAE induction followed by i.p. administration with vehicle or ACDT $(20 \mathrm{mg} / \mathrm{kg}$ ) every day starting from day 1 post immunization. At day 13 or 14 post immunization, mononuclear cells were isolated from the brain and spinal cord of vehicleand ACDT-treated EAE mice. The cell numbers of total $\mathrm{CD}^{+} \mathrm{T}$ cells (A), IFN $\gamma$ - (B), and IL-17expressing $\mathrm{CD}^{+}$Th cells (C) in the brain and spinal cord were determined by FACS analysis. Isotype controls (ISO) were used to determine cells positive for surface expression of CD4 (A) and $\mathrm{CD}^{+} \mathrm{T}$ cells positive for intracellular expression of IFN $\gamma(\mathrm{B})$ or IL-17 (C). Data represent mean \pm SEM. Statistical significance was determined as: $* * * p<0.001$ by unpaired $t$ test.

\section{Fig. 3 ACDT suppresses MG activation}

Primary MG were left untreated (medium) or pretreated with vehicle or ACDT $100 \mu \mathrm{M}$ for 1 hour followed by LPS (100ng/ml) stimulation. (A) 24 hours after LPS treatment, cells were subjected to FACS analysis for surface expression of CD40, CD80, and CD86. Dashed lines represent isotype control. (B) 1.5 and 3 hours after LPS treatment, cells were collected and subjected to RNA extraction followed by Q-PCR analysis for mRNA expression of IL-23p19, 
IL-12p35, IL-12p40, IL-1 $\beta$, IL-6, and GM-CSF. One representative of three independent experiments is shown. Statistical significance was determined as: $* p<0.05$, ** $p<0.01$, and ${ }^{* * *} p<0.001$ by one way ANOVA with post hoc Bonferroni's multiple comparison test. (C) C57BL/6 mice ( $\mathrm{n}=8 /$ group) were subjected to EAE induction followed by i.p. administration with vehicle or ACDT (20mg/kg) every day starting from day 1 post immunization. At day 13 or 14 post immunization, mononuclear cells were isolated from the brain and spinal cord of vehicleand ACDT-treated EAE mice, and the isolated cells were then stained with antibodies of CD45 and CD11b with CD80 or CD86 followed by FACS analysis. MG were determined as $\mathrm{CD} 45^{\text {int }} \mathrm{CD} 11 \mathrm{~b}^{+}$cells, and the expression of CD80 and CD86 on the surface of CD45 ${ }^{\text {int }} \mathrm{CD} 11 \mathrm{~b}^{+}$ cells was determined. Isotype controls (ISO) were used to determine MG positive for CD80 or CD86 expression. Statistical significance was determined as: $* * * p<0.001$ by unpaired $t$ test.

\section{Fig. 4 ACDT lessens neuroinflammation in EAE}

C57BL/6 mice were subjected to EAE induction followed by i.p. administration with vehicle or ACDT $(20 \mathrm{mg} / \mathrm{kg})$ every day starting from day 1 post immunization. (A) At day 13 post immunization, the spinal cord of vehicle- and ACDT-treated EAE mice was harvested and subjected to cytokine array analysis (n=3/group). (B and C) At day 13 or 14 post immunization, the spinal cord and brain of vehicle- and ACDT-treated EAE mice (n=10/group) were harvested and subjected to Q-PCR analysis for mRNA expression of IL-1 $\alpha$, TNF $\alpha$ (B), Ggta1, H2-D1, and Serping1 (C). Statistical significance was determined as: $* p<0.05$, $* * p<0.01$, and $* * * p<0.001$ by unpaired $t$ test.

Fig. 5 ACDT lessens BBB disruption and alleviates spinal cord pathology in EAE 
C57BL/6 EAE animals were i.p. administration with vehicle or ACDT (20mg/kg) every day starting from day 1 post immunization. At day 13 post immunization, vehicle- and ACDT-treated EAE mice ( $n=5 /$ group) were subjected to i.v. administration of Evans blue. (A) 2 hours after Evans blue administration, animals were sacrificed, and the brain and spinal cord of vehicle- and ACDT-treated EAE mice were dissected to assess the leakage of Evans blue. (B) The spinal cord tissues were homogenized and subjected to western blot analysis of MMP3 and MMP9 production. (C and D) The lumbar regions of spinal cord tissues were fixed and subjected to $\mathrm{H} \& \mathrm{E}$ and LFB/cresyl violet staining to assess cell infiltration and demyelination, respectively. The area of cell infiltration and the percentage of demyelination were quantified. Arrow indicates cell infiltration (C) and demyelination (D). Statistical significance was determined as: * $p<0.05$ and $* * p<0.01$ by Mann-Whitney $U$ test.

\section{Fig. 6 ACDT enhances GSH levels in the CNS of EAE}

C57BL/6 EAE animals were i.p. administration with vehicle or ACDT $(20 \mathrm{mg} / \mathrm{kg})$ every day starting from day 1 post immunization. At day 13 post immunization, the spinal card and brain of vehicle- and ACDT-treated EAE animals (n=12/group) were harvested, and GSH levels of spinal cord and brain were determined. Statistical significance was determined as: ${ }^{*} p<0.05$ and $*_{*}^{*} p<0.01$ by unpaired $t$ test. 
Fig. 1

\section{(A)}

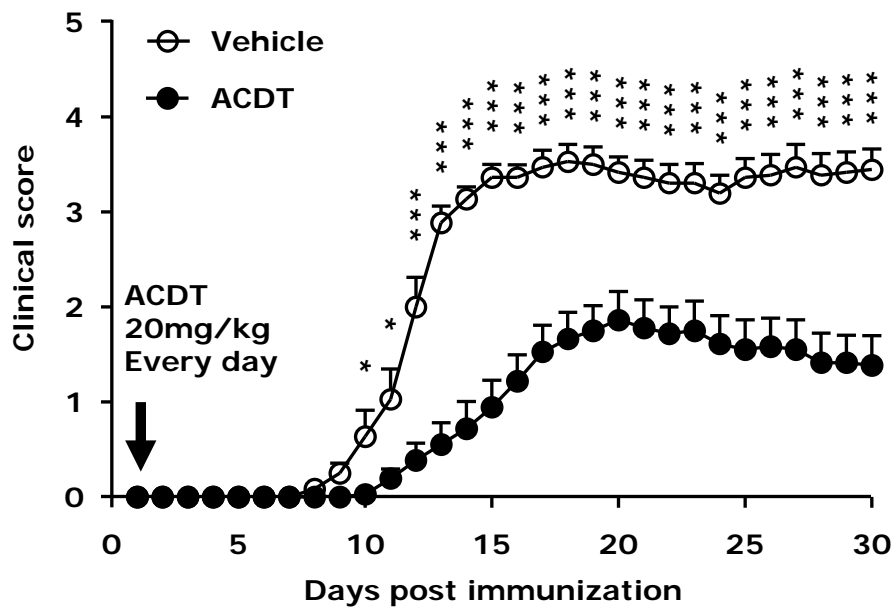

\begin{tabular}{|l|c|c|}
\hline & Vehicle & ACDT $20 \mathrm{mg} / \mathbf{~ k g}$ \\
\hline Incidence & $18 / 18$ & $17 / 18$ \\
\hline Mortality & $2 / 18$ & $0 / 18$ \\
\hline Onset of disease & $10.7 \pm 0.4$ & $14.8 \pm 0.7 * * *$ \\
\hline Maximum score & $4.1 \pm 0.2$ & $2.2 \pm 0.3 * * *$ \\
\hline Cumulative score & $64.3 \pm 2.8$ & $26.6 \pm 4.6 * * *$ \\
\hline
\end{tabular}

(B)

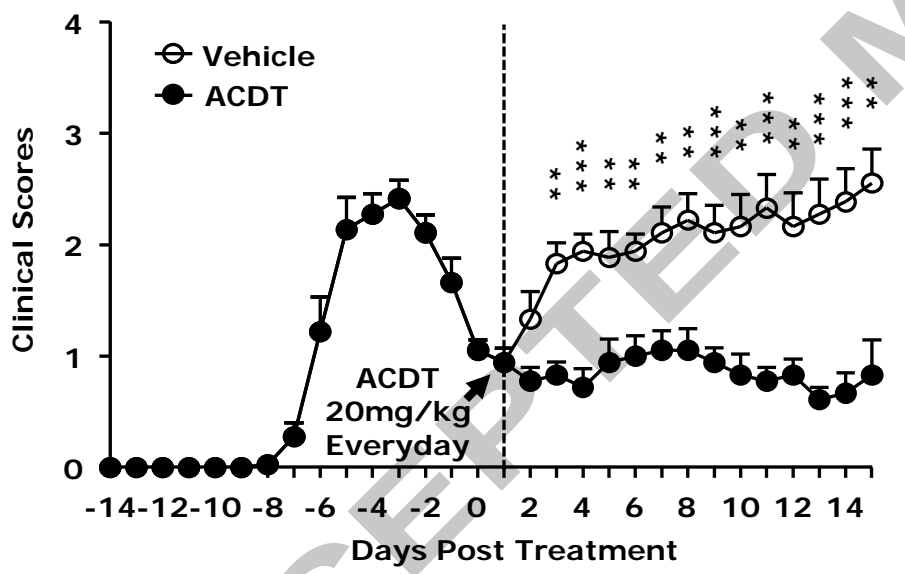

\begin{tabular}{|l|c|c|}
\hline & Vehicle & ACDT 20mg/ kg \\
\hline I ncidence & $9 / 9$ & $9 / 9$ \\
\hline Mortality & $0 / 9$ & $0 / 9$ \\
\hline Onset of disease & $8.8 \pm 0.3$ & $9.3 \pm 0.3$ \\
\hline $\begin{array}{l}\text { Maximum score } \\
\text { post treatment }\end{array}$ & $3.0 \pm 0.3$ & $1.4 \pm 0.2 * *$ \\
\hline $\begin{array}{l}\text { Average score } \\
\text { post treatment }\end{array}$ & $2.0 \pm 0.2$ & $0.8 \pm 0.1 * * *$ \\
\hline $\begin{array}{l}\text { Cumulative score } \\
\text { post treatment }\end{array}$ & $30.2 \pm 2.7$ & $12.6 \pm 1.9 * * *$ \\
\hline
\end{tabular}

Days Post Treatment 
Fig. 2

(A)
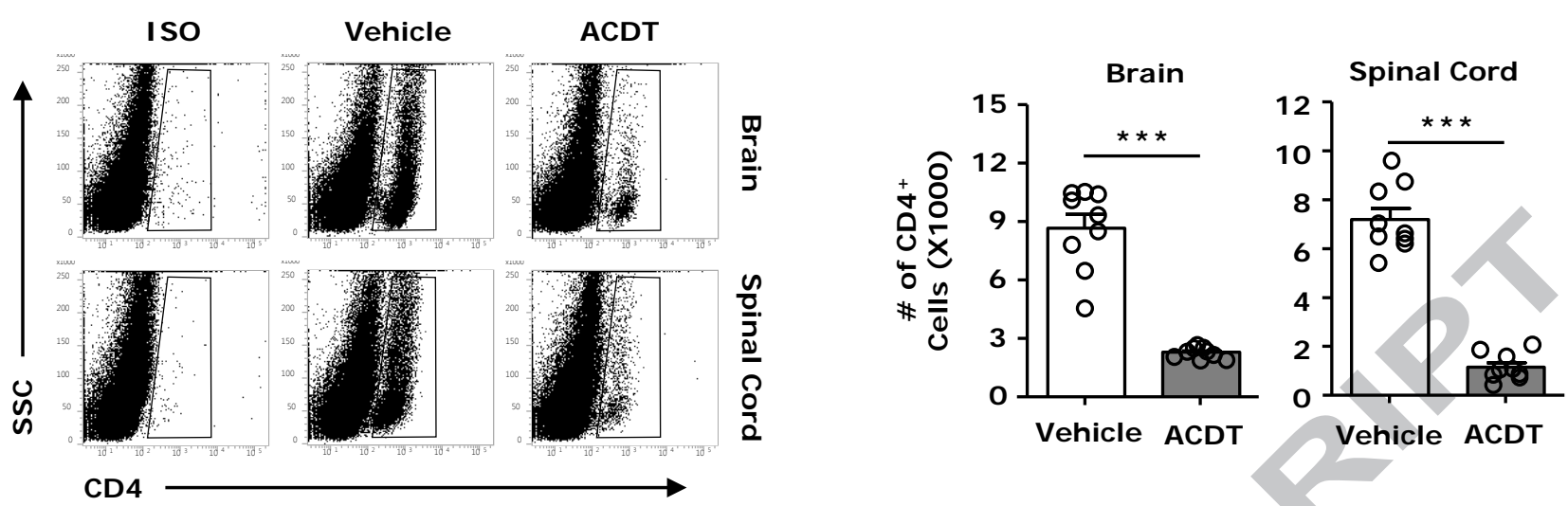

(B)
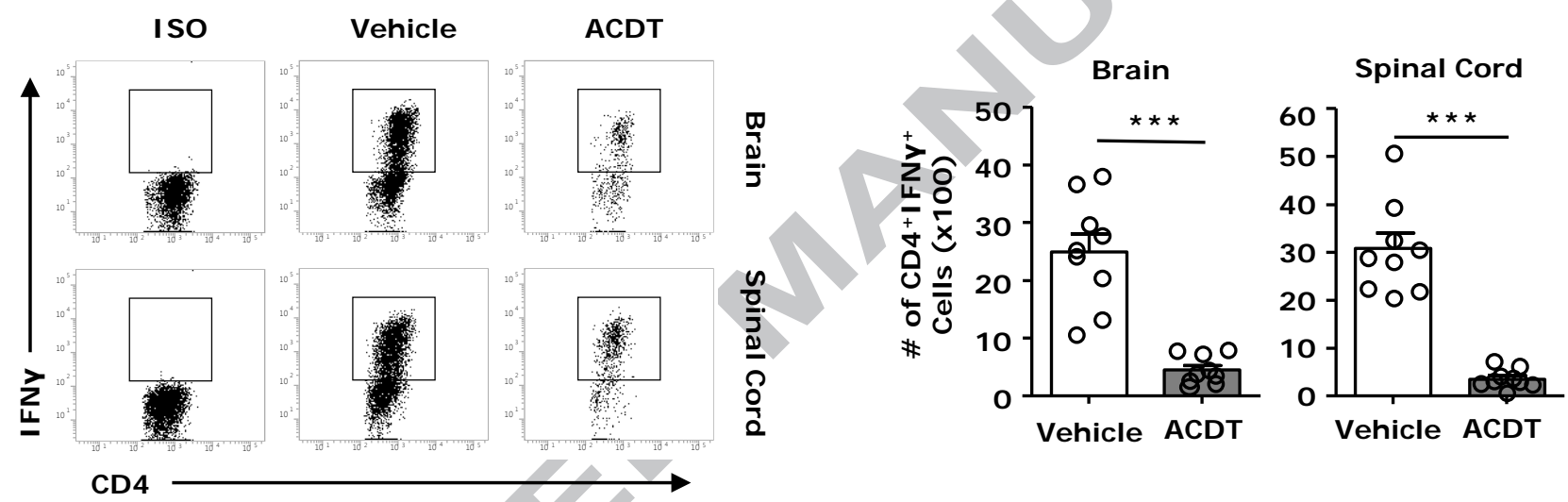

(C)
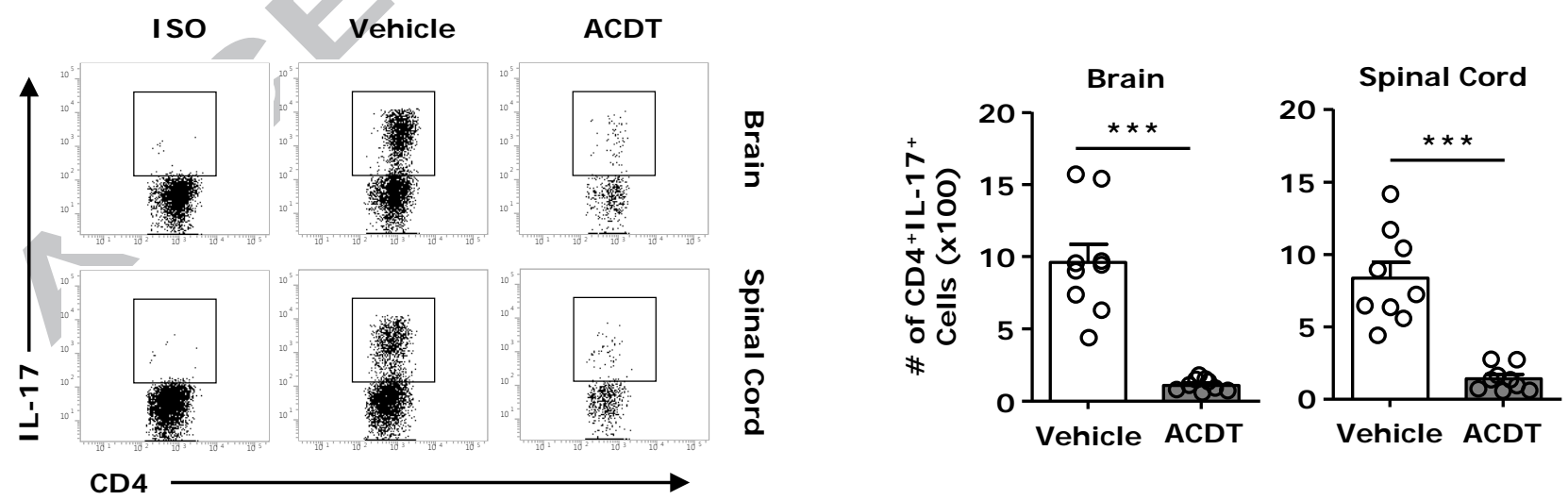
Fig. 3

(A)

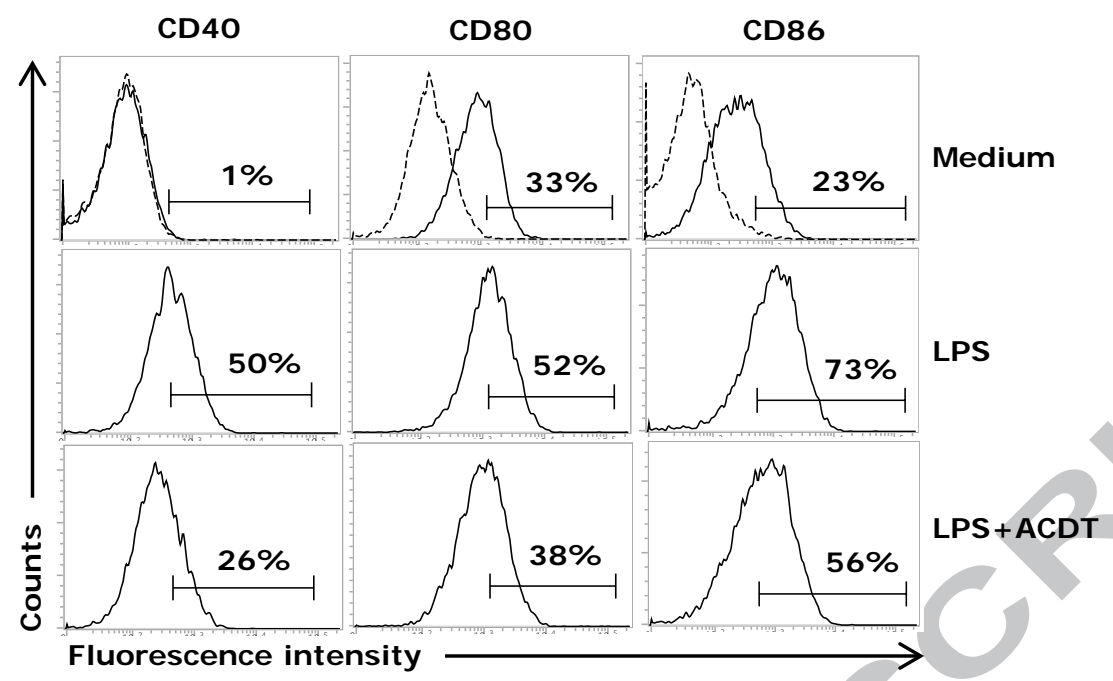

(B)
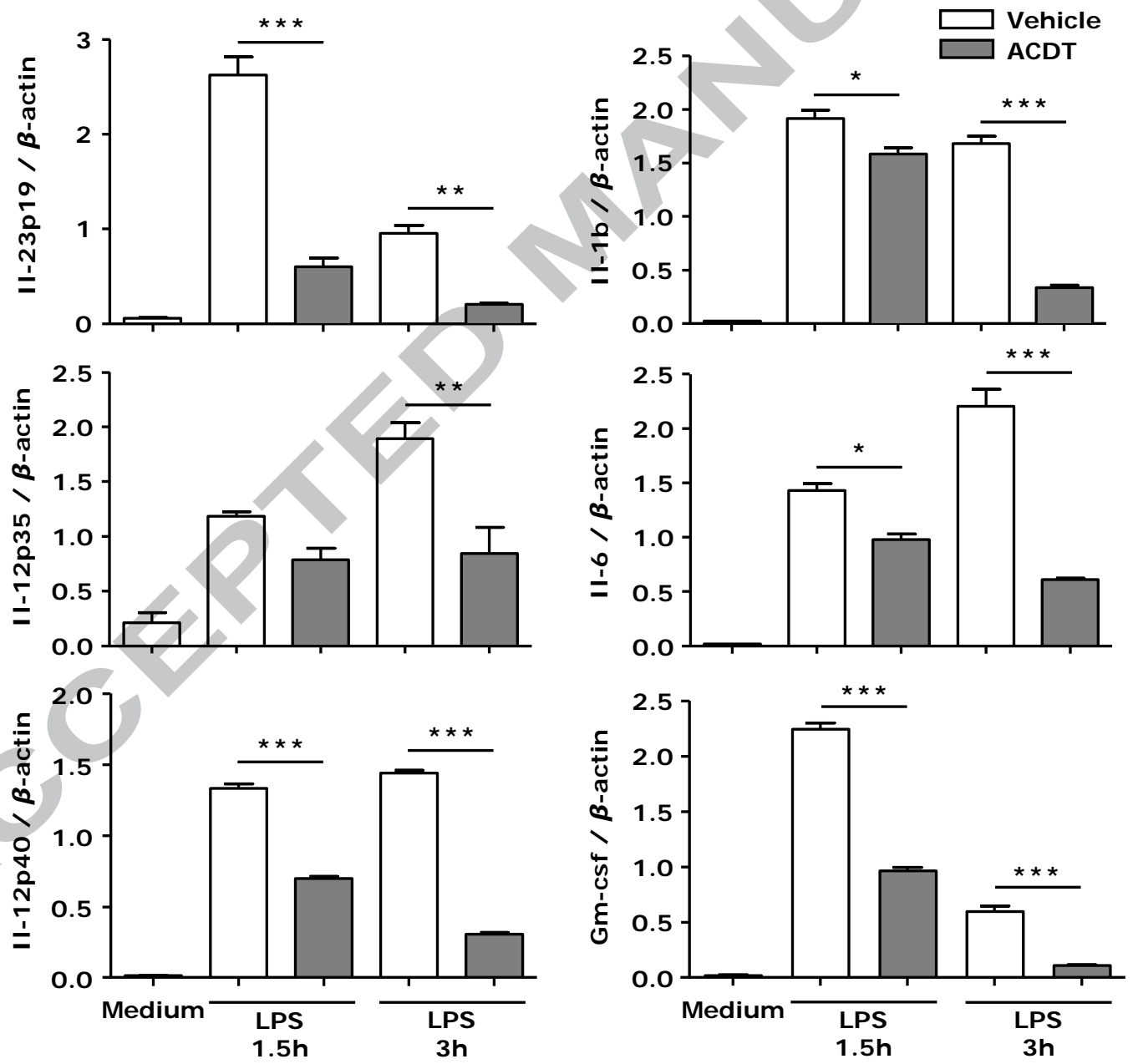
Fig. 3

(C)
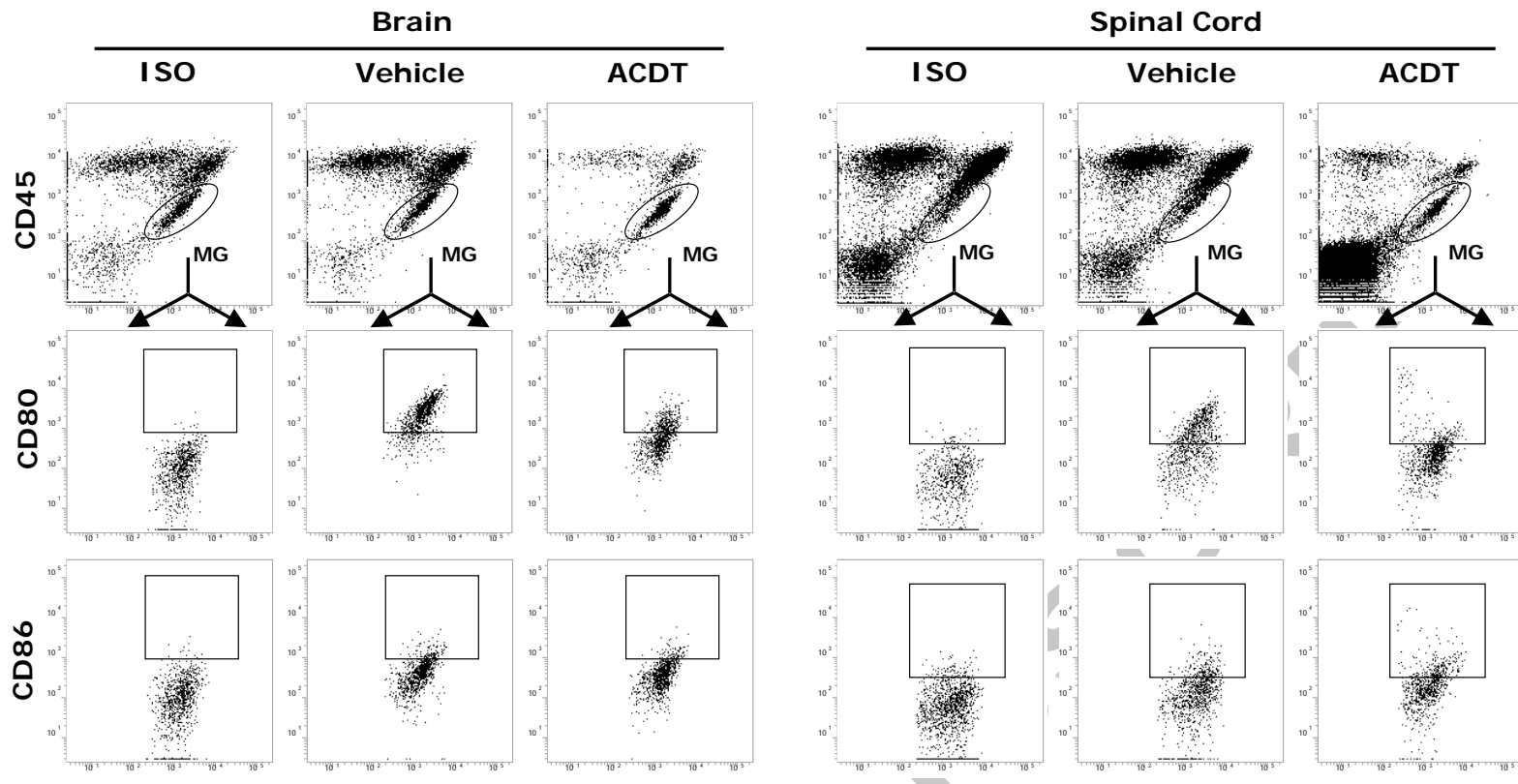

CD11b

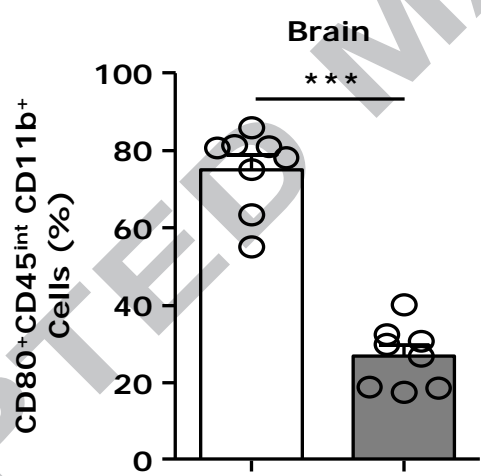

Spinal Cord
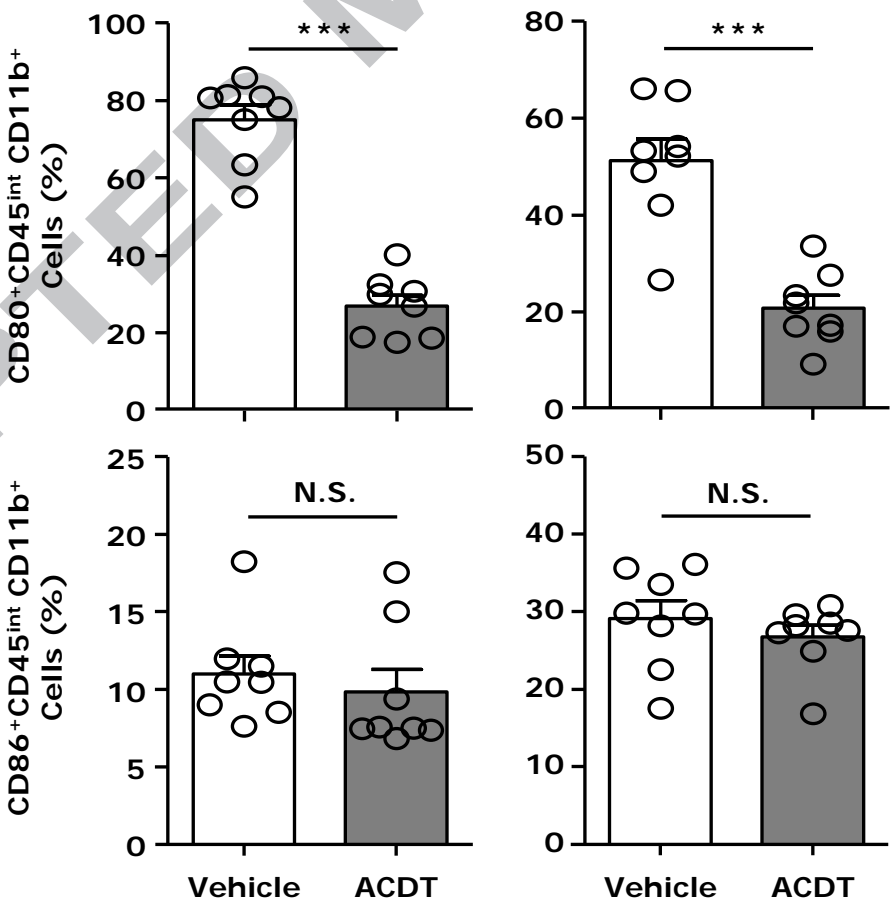
Fig. 4

(A)
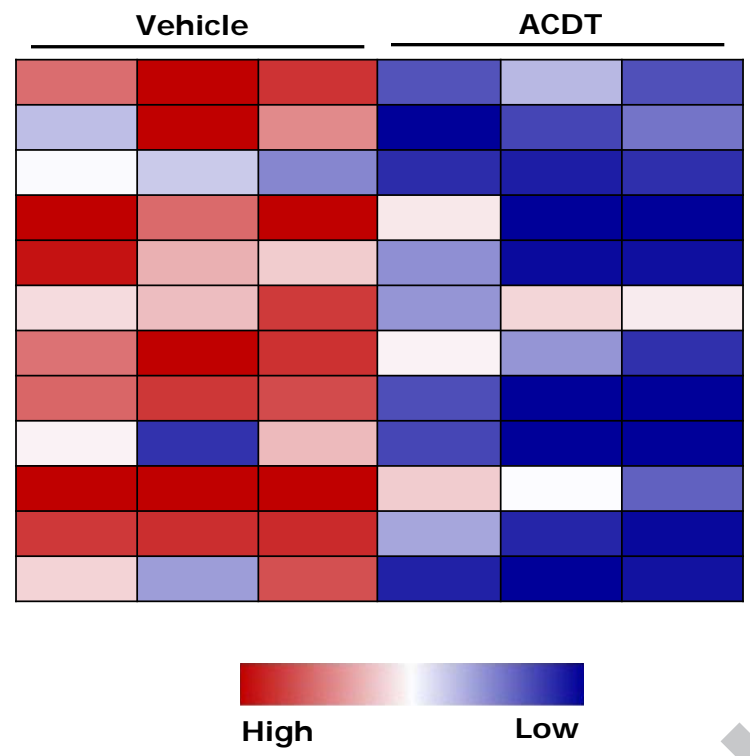

CCL5

CCL6

CCL17

CXCL10

I L-1a

Osteopontin

CD40

Chitinase 3-like 1

C-Reactive Protein I CAM-1

Myeloperoxidase

Pentraxin 3

(B)

(C)
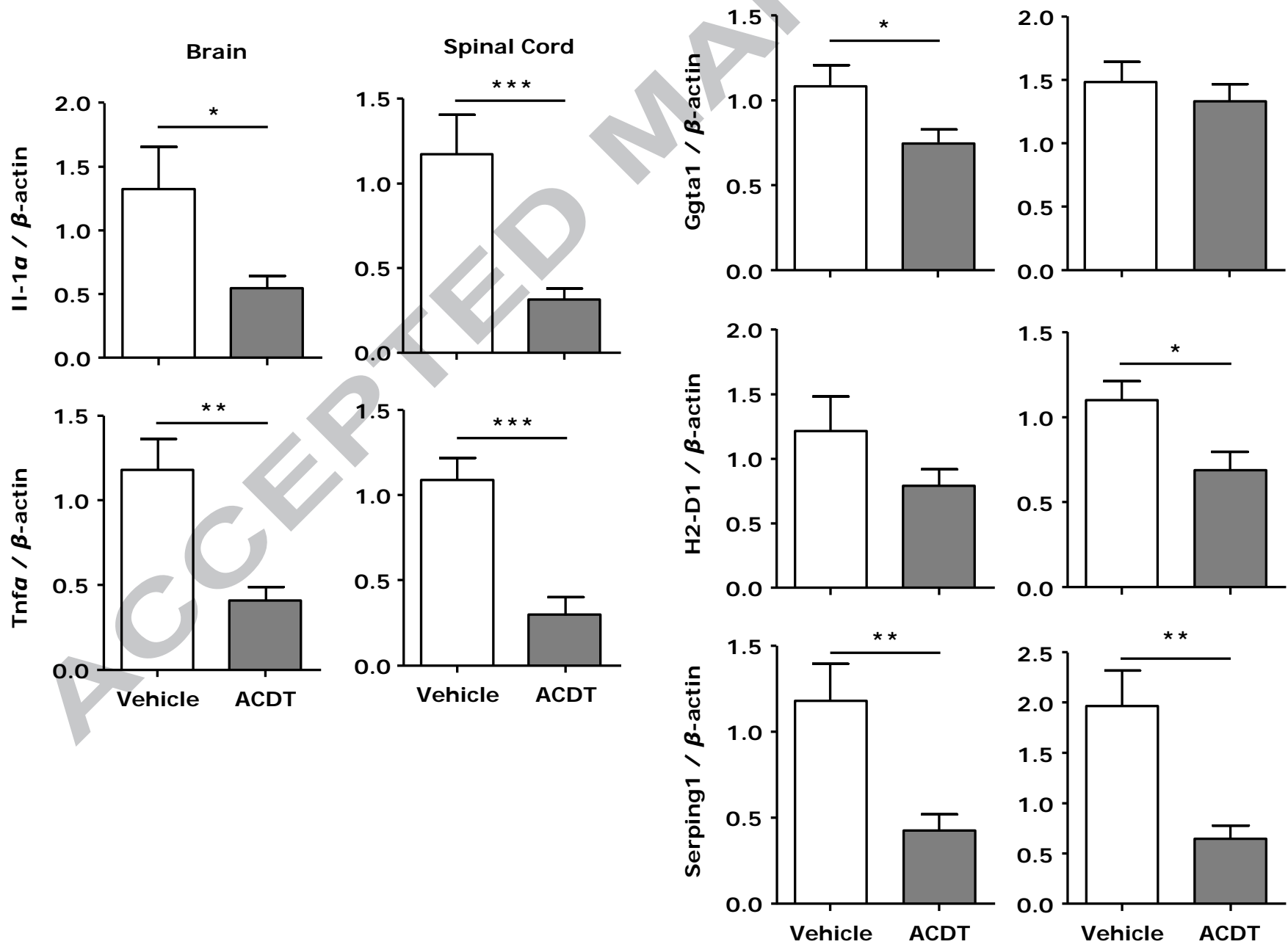
Fig. 5 (A)

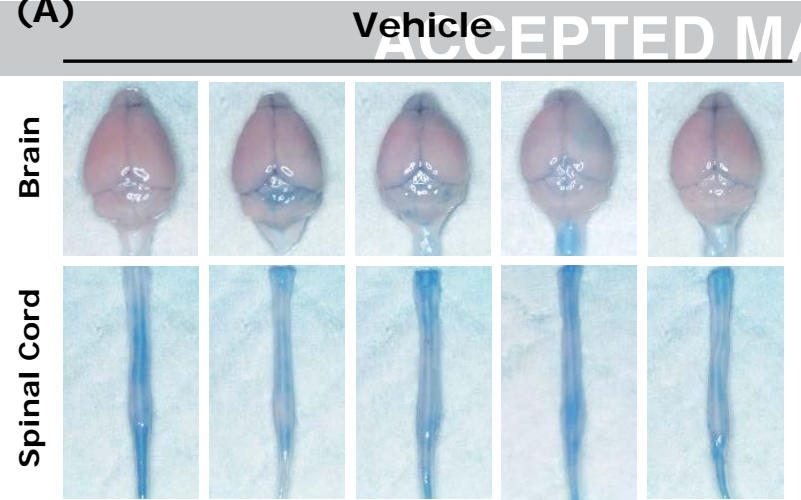

(B)
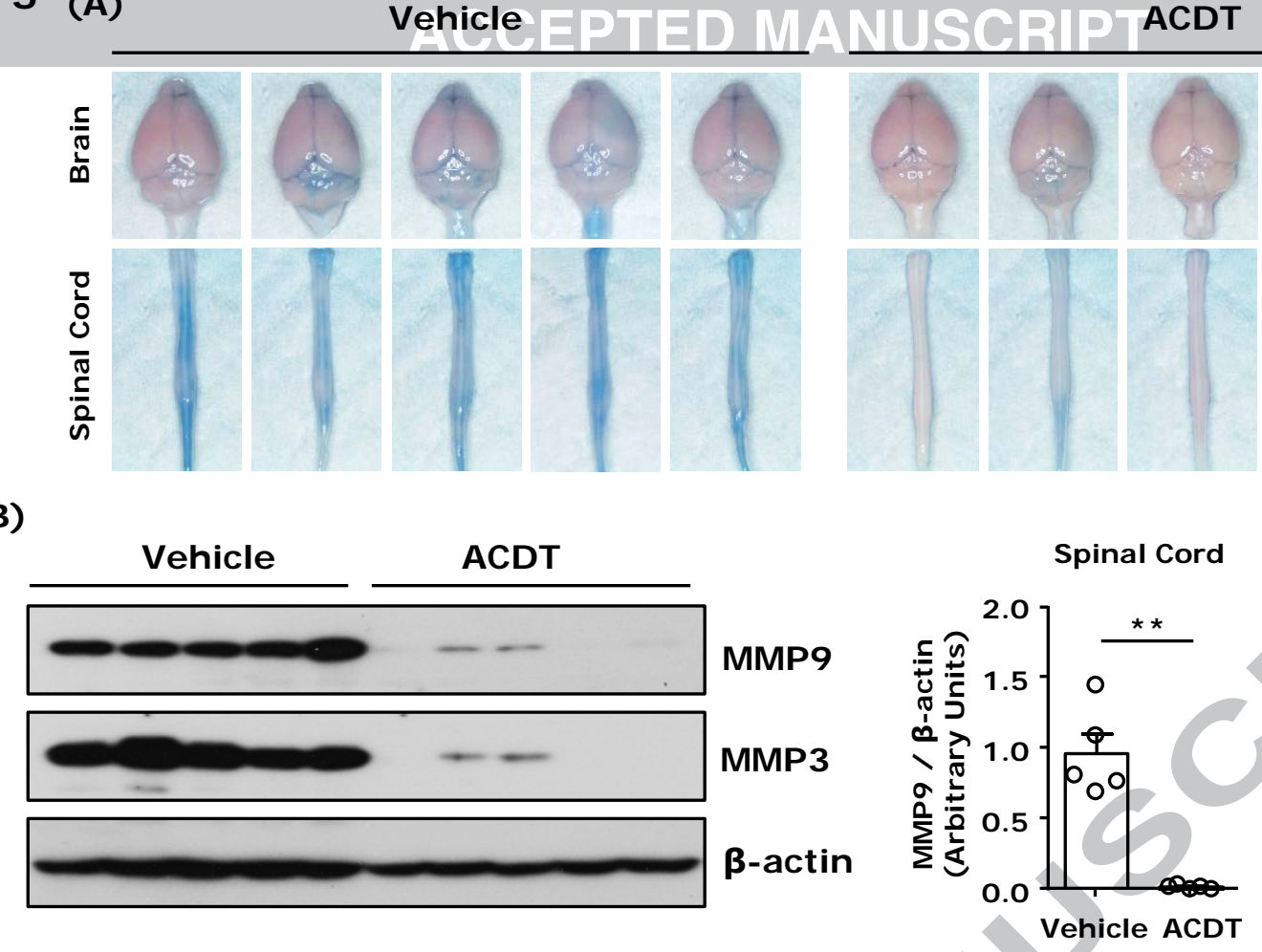

(C)
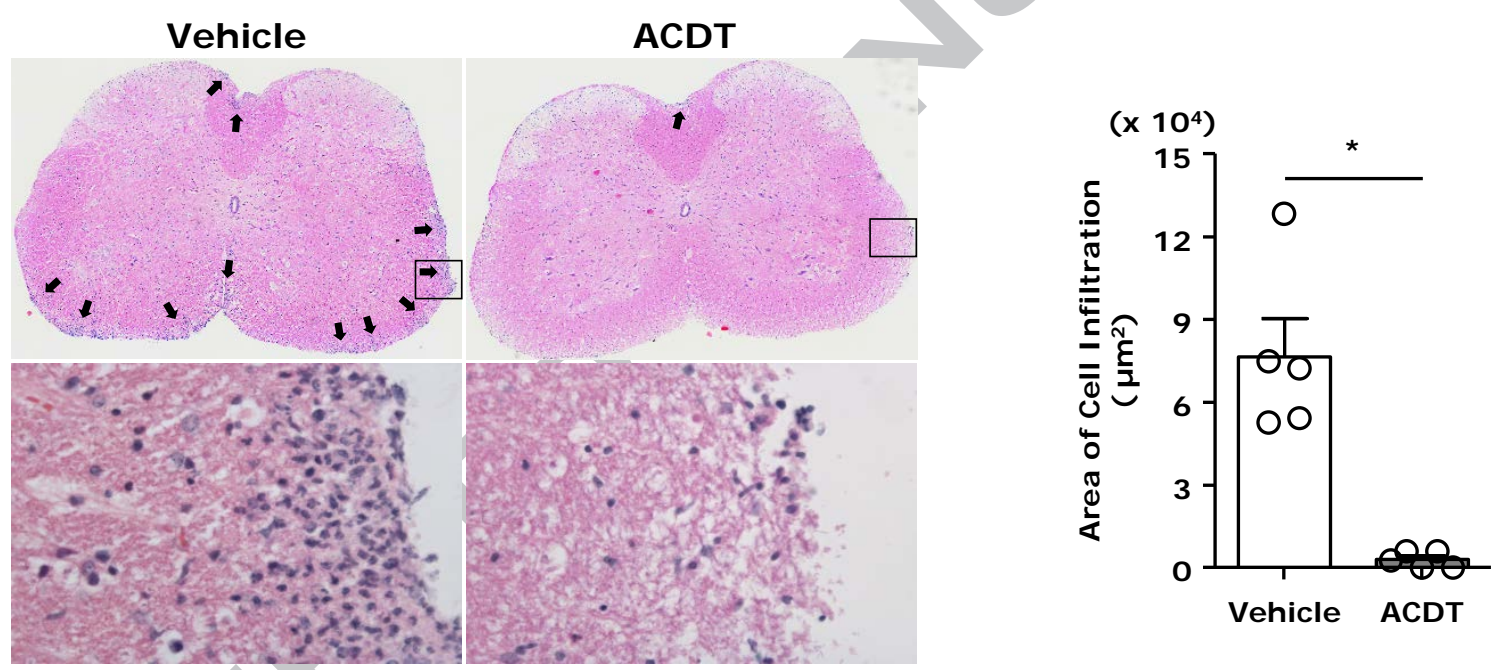

(D)
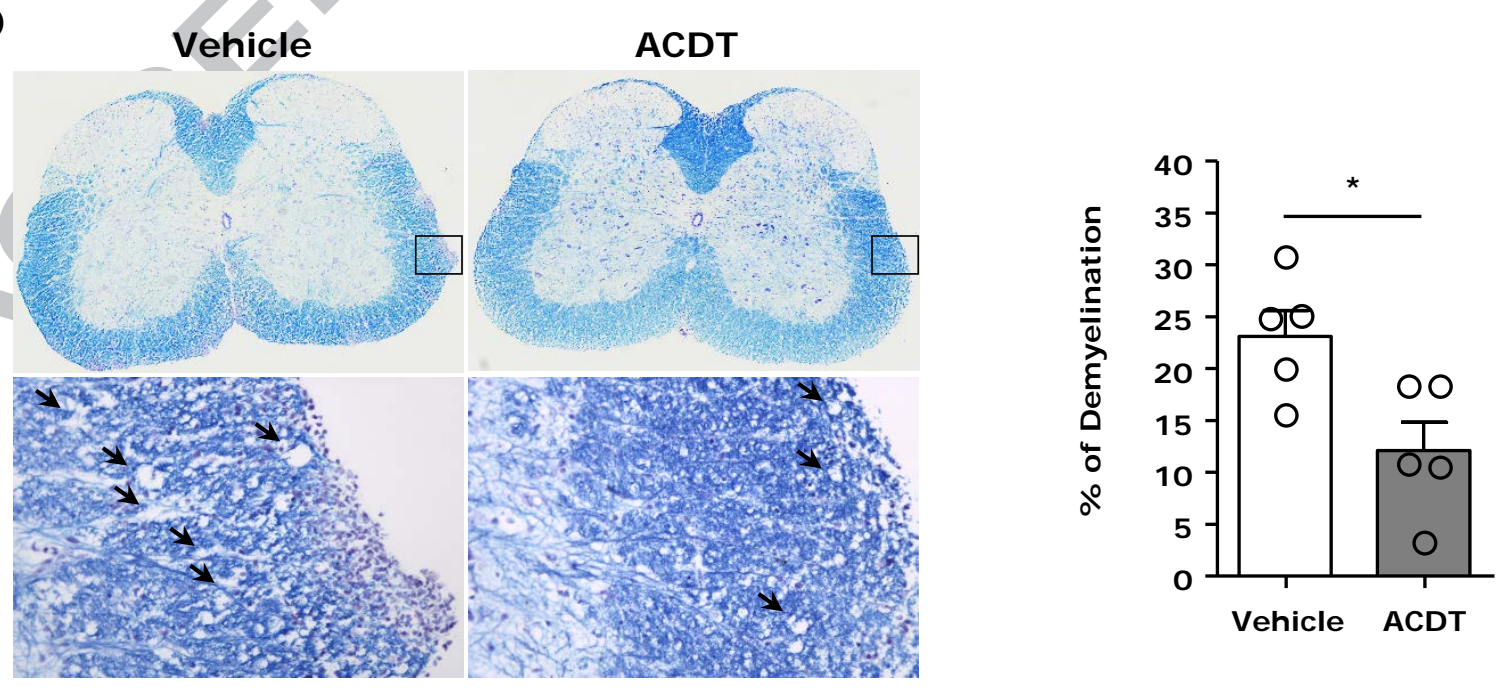
Fig. 6

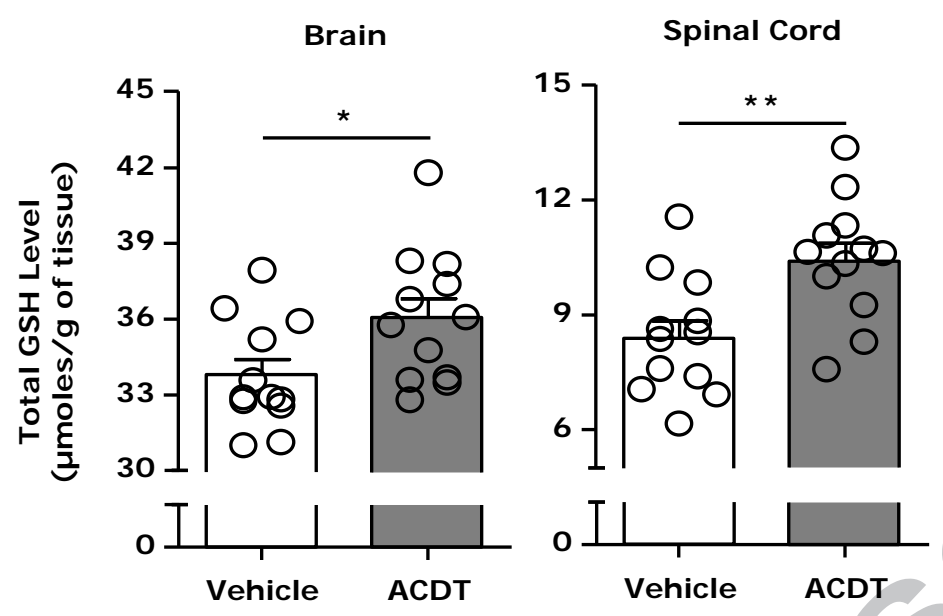




\section{Highlights}

- Dithiolethione ACDT ameliorates disease severity in both chronic and relapsing-remitting EAE.

- ACDT inhibits the CNS infiltration of pathogenic T cells in EAE.

- ACDT suppresses MG activation in vitro and in vivo.

- ACDT alleviates neuroinflammation and reduced neurotoxic A1 astrocyte generation in EAE.

- ACDT lessens blood-brain barrier disruption and alleviates spinal cord pathology in EAE. 